Recepción: 20 / 04 / 2017

Aceptación: 20 / 05 / 2017

Publicación: 15 / 07 / 2017

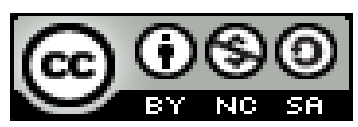

Ciencias técnicas y aplicadas Artículo Científico

\title{
Determinación y Optimización del punto de operación de las bombas del sistema electro-sumergible para pozos petroleros
}

\section{Determination and Optimization of the point of operation of the pumps of the system electro-submersible for oil wells}

\section{Determinação e otimização do ponto de operação das bombas do sistema eletro- submersível para poços de petróleo}

\section{Edison R. Suntaxi-Guanotoa ${ }^{I}$ \\ edisao5@hotmail.com}

Rene A. Carrillo-Flores II

rcarrillo@uce.edu.ec

Iván C. Naula-Reina ${ }^{\text {III }}$

icnaula@uce.edu.ec

\author{
Carlos F. Izurieta-Cabrera IV \\ cizurieta@uce.edu.ec \\ Guillermo A. Albuja-Proaño v \\ galbuja@uce.edu.ec \\ Darwin R. Caina-Aysabucha VI \\ drcaina@uce.edu.ec
}

Correspondencia: edisao5@hotmail.com

\footnotetext{
I. Magister en Procesos Industriales; Diploma Superior en Redes Digitales Industriales; Ingeniero de Ejecución en Electrónica e Instrumentación; Andes Petroleum Ecuador Ltd, Universidad Central del Ecuador, Quito, Ecuador.

II. Magister en Ingeniería Industrial; Ingeniero en Informática; Universidad Central del Ecuador, Quito, Ecuador.

III. Magister en Estadística Aplicada; Ingeniero Informático; Ingeniero Matemático; Universidad Central del Ecuador, Quito, Ecuador.

Iv. Magister en Docencia Universitaria en Ciencias de la Ingeniería; Ingeniero Matemático; Universidad Central del Ecuador, Quito, Ecuador.

v. Magister en Matemáticas Puras y Aplicadas; Ingeniero Matemático; Ingeniero Informático; Universidad Central del Ecuador, Quito, Ecuador.

VI. Ingeniero en Electrónica y Telecomunicaciones; Magister en Ciencias en Tecnologías de la Información; Universidad Central del Ecuador, Quito, Ecuador.
} 


\section{Resumen}

Esta investigación está orientada a realizar la optimización del punto de operación de las bombas electro-sumergibles de pozos petroleros, aplicando una de las técnicas más utilizadas para optimizar sistemas de producción debido a su comprobada efectividad y confiabilidad a nivel mundial como es el Análisis Nodal. Esta técnica consiste en realizar varios análisis de sensibilidad de las variables más importantes y cuantificar su impacto sobre la producción. Las variables que mayor impacto tienen son las que causan restricciones al sistema; por lo tanto, las oportunidades de mejora se las busca tanto en variables de salida, como de entrada, para finalmente seleccionar la mejor opción desde el punto de vista técnico y sin descuidar bajo ningún concepto el factor económico que involucra.

Palabras claves: Bomba electro-sumergible; SubPUMP; optimización; análisis nodal; petróleo. 


\begin{abstract}
This investigation is focused on the optimization of the operation point of the electrical -submersible oil well pumps, the optimization technique utilized to optimize production systems is the nodal analysis, because of the proven effectiveness and reliability worldwide. This technique involves making several sensitivity analysis of the most important variables and quantify their impact on production. The variables that have the greatest impact are those that cause restrictions to the system; thus improvement opportunities will be found in both output variables and input variables, to finally select the best technical and economical option.
\end{abstract}

Key words: Electrical submersible pump; SubPUMP; optimization; nodal analysis; oil. 


\section{Resumo}

Esta investigação está focada na otimização do ponto de operação das bombas de poços de petróleo elétrica, a técnica de otimização utilizada para otimizar os sistemas de produção é a análise nodal, devido à comprovada eficácia e confiabilidade em todo o mundo. Esta técnica envolve a realização de várias análises de sensibilidade das variáveis mais importantes e quantifica seu impacto na produção. As variáveis que têm o maior impacto são aquelas que causam restrições ao sistema; Assim, as oportunidades de melhoria serão encontradas nas variáveis de saída e nas variáveis de entrada, para finalmente selecionar a melhor opção técnica e econômica.

Palavras-chave: Bomba elétrica submersível; Sump PUMP; Otimização; Análise nodal; óleo. 


\section{Introducción.}

El bombeo electro-sumergible es un sistema integrado de levantamiento artificial, es considerado como un medio económico y efectivo para levantar altos volúmenes de fluido desde grandes profundidades en una variedad de condiciones de pozo (Gabor 2009; Baker Hughes 2009). Es más aplicable en yacimientos con altos porcentajes de agua y baja relación gas-aceite; sin embargo, en la actualidad estos equipos han obtenido excelentes resultados en la producción de fluidos de alta viscosidad, en pozos gasíferos, en pozos con fluidos abrasivos, en pozos de altas temperaturas y de diámetro reducido (Sachdeva, 1988).

Los componentes del sistema de bombeo electro-sumergible pueden ser clasificados en dos partes, el equipo de fondo y el equipo de superficie. El equipo de fondo cuelga de la tubería de producción y cumple la función de levantar la columna de fluido necesaria para producir el pozo, consiste principalmente de un motor eléctrico, un sello, un separador de gas y una bomba electro centrífugo. Un cable de poder transmite la energía eléctrica de la boca del pozo hasta el motor. El equipo de superficie provee de energía eléctrica al motor electro-sumergible y controla su funcionamiento. Los principales componentes de superficie son los transformadores, el controlador de velocidad o variador de frecuencia, y la caja de venteo. Varios componentes adicionales normalmente incluyen la cabeza de pozo, válvulas de retención y de drenaje, entre otros. Lo anteriormente descrito, se puede apreciar en la figura 1. El equipo electro-sumergible puede incluir un sensor de presión de fondo de pozo y temperatura para observar las condiciones en la cavidad del pozo. 
Edison R. Suntaxi-Guanotoa; Rene A. Carrillo-Flores; Iván C. Naula-Reina; Carlos F. Izurieta-Cabrera; Guillermo A. AlbujaProaño; Darwin R. Caina-Aysabucha

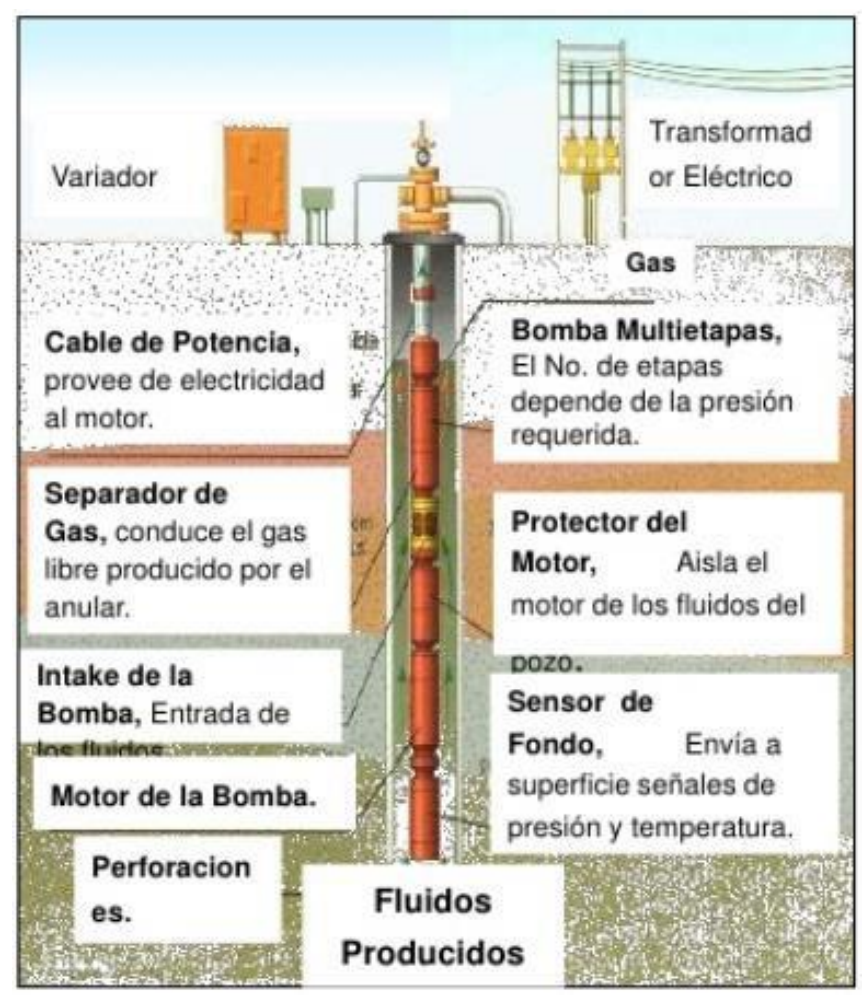

Figura 1. Sistema de bombeo electro-sumergible (Instipetrol, 2017)

El sistema de Análisis nodal (Beggs 2003; Maggiolo 2008), se utiliza para evaluar el rendimiento de los pozos de producción o inyección. Esto implica especificar un punto nodal, en el fondo del pozo o en su la cabeza (ver figura 2a), y dividir el sistema de producción en dos partes: entrada y la salida (figura 2b). Todo el sistema se representa en la figura 2.

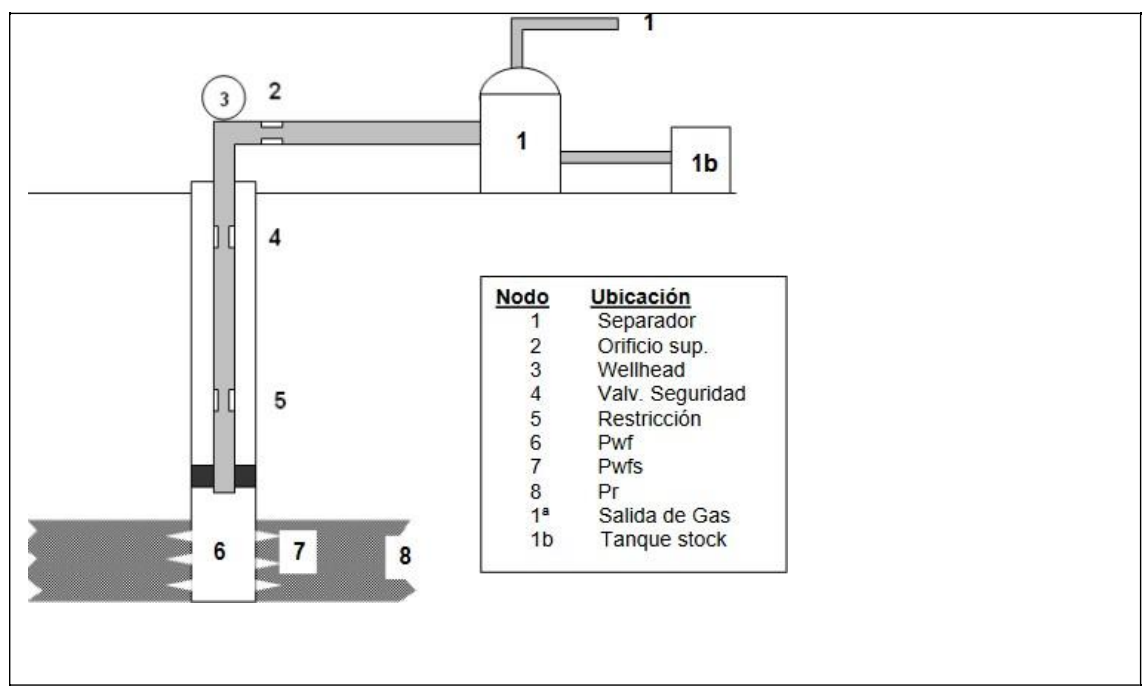




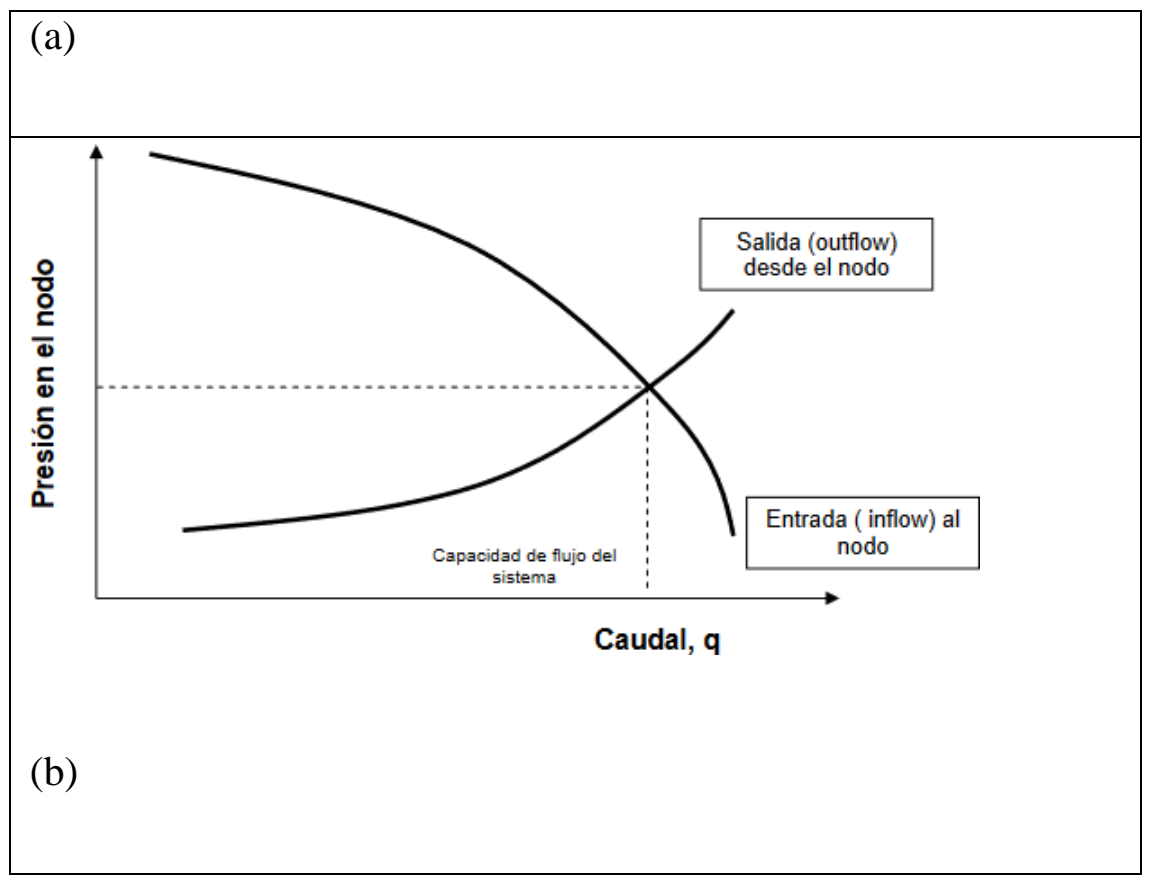

Figura 2. Sistema de análisis nodal (Oilproduction, 2017)

El nodo de la solución se define como la ubicación donde el diferencial de presión aguas arriba (flujo de entrada) y aguas abajo (salida) del nodo es cero. El nodo solución se pueden seleccionar cuidadosamente para aislar el efecto de ciertas variables. Por ejemplo, si el nodo es escogido en el fondo del pozo, factores que afectan el rendimiento del flujo de entrada en un pozo productor, como el factor de daño de formación, puede ser analizado independientemente de las variables que afectan el flujo de salida, tales como el diámetro de la tubería o la presión del separador.

Se utiliza el nodo en la descarga de la bomba, lo cual implica tener dos ecuaciones que igualadas permiten obtener el punto óptimo de operación del sistema: ecuación general de la bomba centrifuga más yacimiento y la ecuación general de la energía entre la cabeza del pozo y el final de la tubería. 
Para encontrar la curva de rendimiento del primer subsistema, partimos de la presión de entrada a la bomba, PIP, y añadir el aumento de presión desarrollada por la bomba para encontrar la presión disponible en la descarga de la misma:

$$
P_{d}=P I P+\Delta P_{p}
$$

Para encontrar la presión fluyente del pozo, Pwf, e ir relacionando con los parámetros de yacimiento aplicamos la siguiente formula:

$$
P_{w f}=P I P+\left(L_{p e r f}-L_{p u m p}\right) \operatorname{grad}_{l}
$$

Con esta presión de fondo fluyente se puede calcular el índice de productividad del pozo IP, el cual es función de los parámetros de yacimiento, e indica el aporte o potencial del pozo de petróleo (bopd/psi).

$$
\begin{gathered}
I P=\frac{Q}{P_{y a c}-P_{w f}} \\
I P=\frac{0.00708 \frac{k h}{r_{e}}}{\mu B \ln \frac{\left(\frac{r_{w}}{r_{w}}\right.}{r}}
\end{gathered}
$$


Determinación y Optimización del punto de operación de las bombas del sistema electro-sumergible para pozos petroleros

La curva de rendimiento de la sarta de tubería representa la presión de descarga requerida por la bomba ESP y se calcula con la presión de cabeza de pozo, el peso de la columna de fluido y la suma de las pérdidas de presión en la tubería.

$$
P_{d}=W H P+L_{p u m p} \operatorname{grad}_{l}+\Delta P_{f r}
$$

Ya que la presión disponible y la presión requerida deben ser igual en el nodo solución, la solución simultánea de las dos fórmulas para el incremento de presión será desarrollada por la bomba ESP obteniendo en la siguiente expresión:

$$
\Delta P_{p}=W H P+L_{p u m p} \operatorname{grad}_{l}+\Delta P_{f r}-P I P
$$

Dónde:

$F B H P=$ presión de fondo fluyente, psi

$\operatorname{Grad}_{l}=$ gradiente de líquido, $\mathrm{psi} / \mathrm{ft}$

$\Delta P_{\text {pump }}=$ incremento de presión desarrollada por la bomba, psi

$L_{s e t}=$ profundidad de ajuste de la bomba, $\mathrm{ft}$

$L_{p e r f}=$ profundidad de las perforaciones, $\mathrm{ft}$

Las pérdidas por fricción se calculan con la ecuación usada por Hazen-Williams.

$$
\Delta P_{f r}=0.2083\left(\frac{100}{C}\right)^{1.85} \frac{q^{1.85}}{I D^{4.86}}
$$

Dónde:

$\Delta h_{f}=$ perdida de carga por fricción, ft/100ft

$C=$ coeficiente de calidad de tubería

$q=$ cantidad de flujo, gpm 
$I D$ = diámetro interno de la tubería, in.

\section{Eficiencia de potencia en sistema ESP.}

La potencia consumida por el motor ESP comprende, además de la energía necesaria para levantar los fluidos del pozo a la superficie, todas las pérdidas de energía de los equipos de fondo y superficie.

\section{Potencia en el Sistema ESP.}

En las instalaciones de ESP, el trabajo útil de salida es hecho por la bomba centrífuga cuando se levanta una cantidad de líquido de la profundidad de asentamiento de la bomba a la superficie. Este trabajo es llamado potencia hidráulica útil, y se puede calcular como la potencia consumida para incrementar el potencial de la energía del líquido bombeado.

$$
P_{h y d r}=1.7 \times 10^{-5} q_{l}\left(0.433 \gamma_{l} L_{p u m p}-P I P\right)
$$

Dónde:

$P_{h y d r}=$ potencia hidráulica para elevación del líquido, Hp

$q_{l}=$ cantidad de líquido producido, bpd

$\gamma_{l}=$ gravedad específica del líquido producido

$L_{\text {pump }}=$ profundidad de la bomba, $\mathrm{ft}$

$P I P=$ presión de succión de la bomba, psi

La entrada de energía eléctrica a partir de la fuente de alimentación de la superficie, Pe, cubre el requerimiento de energía de la instalación ESP, incluyendo la potencia útil usada para levantar el fluido y las pérdidas de energía ocurridas en el equipo de fondo y superficie. 
Es usualmente medida por un medidor de potencia de tres fases en el sitio o bien se puede calcular del requerimiento de $\mathrm{kVA}$ del sistema y el factor de potencia:

$$
P_{e}=1.732 \times 10^{-3} I U_{\text {surf }} \cos \emptyset
$$

Dónde:

$P_{e}=$ potencia eléctrica total de entrada, $\mathrm{KW}$

$U_{\text {surf }}=$ voltaje de superficie requerido por el sistema, voltios

$I=$ corriente del motor, amperios

$(\cos \varnothing)_{\text {system }}=$ factor de potencia del sistema ESP

El rendimiento del sistema se puede calcular relacionando el sistema de potencia útil (potencia hidráulica gastada en levantar el fluido) y la entrada total de energía (potencia eléctrica requerida en superficie).

$$
\eta_{\text {system }}=\frac{P_{h y d r}}{\frac{P_{e}}{0.746}}=0.746 \frac{P_{h y d r}}{P_{e}}
$$

Dónde:

$P_{h y d r}=$ potencia hidráulica para elevación del líquido, Hp

$P_{e}=$ potencia eléctrica total de entrada, $\mathrm{KW}$ 


\section{Materiales y métodos.}

La investigación utiliza el programa SubPUMP que ayuda a realizar el análisis del desempeño de las bombas electro-sumergibles. Una de las principales aplicaciones de este programa es simular la operación de las bombas electro-sumergibles, lo cual me va permitir realizar múltiples balances con diferentes valores de las variables que intervienen y luego cuantificar el impacto que tiene dicha variable sobre la operación de las bombas electro-sumergibles.

Con el fin de optimizar el bombeo electro-sumergible se realiza una serie de análisis donde se predice el comportamiento actual y futuro de un pozo productor.

La aplicación del análisis Nodal contribuirá a determinar el tamaño y la potencia mínima requerida por la bomba para enviar el fluido del yacimiento a la superficie, observando el comportamiento y cambio de potencia a diferentes valores de variables.

Para efectuar un análisis nodal en una bomba electro-sumergible comenzamos seleccionando un nodo en la bomba, donde esta pueda manejarse como un componente independiente en el sistema.

\section{Resultados y discusiones.}

En esta sección encontrara algunos resultados importantes sobre todo del simulador, corridas del programa con una comparación entre las soluciones exactas versus las aproximadas, así como también graficas de en 2D y 3D del proceso de infiltración.

Presentamos la pantalla del programa computacional que resuelve la ecuación de Richards bidimensional, se presenta las soluciones aproximadas y exactas en los nodos de la malla para lo cual se ha usado un problema cuya solución se conoce. 


\section{Diseño del sistema con BES}

Se realiza el análisis del diseño de un pozo petrolero el mismo que fue hecho en condiciones iniciales con datos y características referenciales obtenidos de resultados de registros eléctricos realizados en el pozo, así como con datos de pruebas obtenidas del yacimiento, o de otros pozos cercanos al yacimiento, estos datos de diseño por lo general son los siguientes: Diámetro del casing, diámetro y características del tubing, profundidad de los disparos, presión de cabeza del tubing (THP), presión de cabeza del casing (CHP), tasa de producción deseada, nivel de fluido dinámico y presión de intake de la bomba (Pwf), nivel de fluido estático, relación Gas/Petróleo (GOR), corte de agua (BSW), índice de Productividad (IP).

El análisis describe que todas características del pozo juegan un papel importante y crítico en el diseño adecuado de un sistema de bombeo eléctrico-sumergible (ESP); pues afectan directamente al rendimiento, a la eficiencia y a la longevidad del sistema ESP debido a que si estos datos difieren demasiado de los datos obtenidos después de que el pozo se haya estabilizado y se obtengan datos reales con pruebas de producción y análisis del fluido, va ser difícil que la bomba electro-sumergible trabaje eficientemente y por lo tanto podría causar un daño prematuro en el mismo. Sin embargo, si estos datos no difieren demasiado se podría lograr que la bomba trabaje eficientemente variando algún parámetro que se pueda controlar y de esta manera optimizar el desempeñó y trabajo del sistema electro-sumergible.

Debido a que la bomba electro-sumergible es poco flexible cuando opera a una velocidad fija, el equipo está limitado a un caudal de producción y a una altura de columna dinámica generada fija. En este diseño se utiliza un variador de frecuencia VSD, el cual es como un accesorio del sistema de bombeo electro-sumergible de gran valor que ayuda eliminar estas restricciones, permitiendo variar la velocidad del equipo y con esto se puede cambiar el caudal y/o la altura de 
columna dinámica dependiendo de las aplicaciones. Estos cambios se logran únicamente cambiando la velocidad de operación, sin tener que modificar el equipo de fondo.

En la figura 3, se puede observar las curvas realizadas con las formulas anteriores mencionadas. La curva de "outflow", es la presión necesaria que requiere la bomba para levantar una cantidad de petróleo y llevarlo hasta las facilidades de proceso y la curva "inflow", es la presión que el yacimiento aporta para levantar el fluido a determinada profundidad, en este caso se lo utilizara en la entrada de la bomba. La diferencia de estas dos curvas a cualquier caudal es la presión o energía que debe de aportar la bomba para levantar el fluido desde el subsuelo.

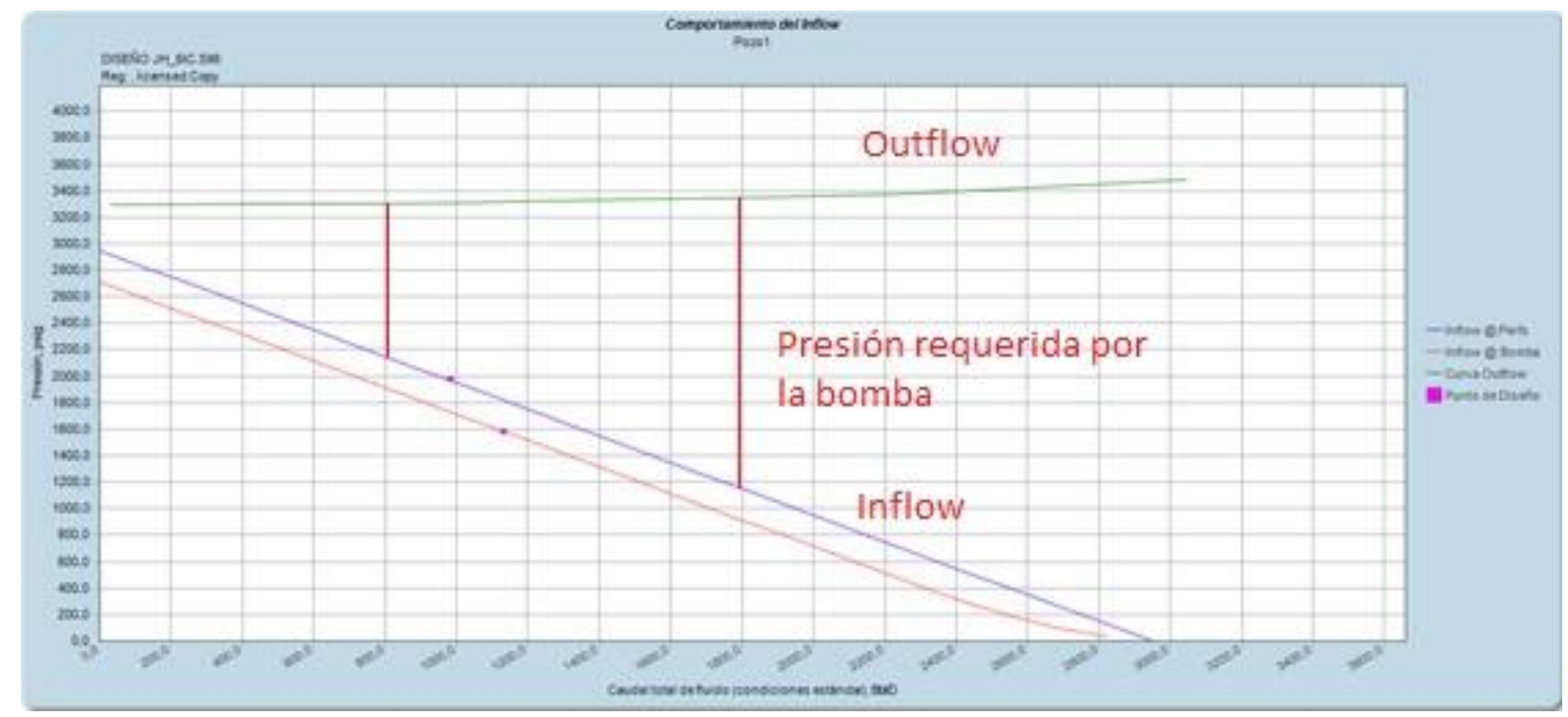

Figura 3. Curva de rendimiento del pozo con levantamiento artificial (Derechos de Autor - Resultados de Análisis SubPUMP)

En la siguiente curva de la figura 4, se observa la intersección de la curva de la bomba con la curva del sistema, la intersección de las curvas es el punto óptimo de operación de los dos sistemas. 
Determinación y Optimización del punto de operación de las bombas del sistema electro-sumergible para pozos petroleros

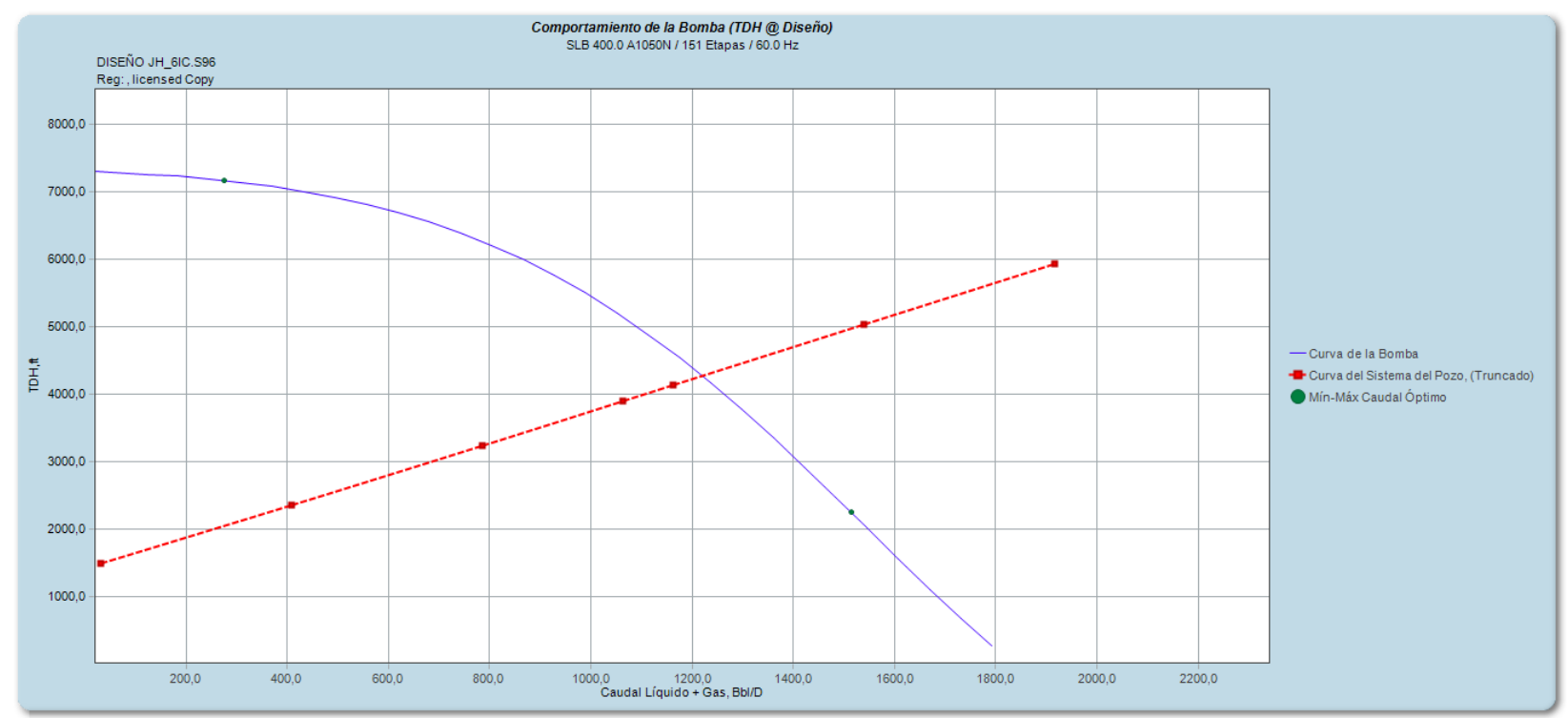

Figura 4. Curva de la bomba con el sistema

(Derechos de Autor - Resultados de Análisis SubPUMP)

Un sistema de levantamiento por bombeo eléctrico sumergible tiene una bomba centrifuga multietapas, la cual puede variar su velocidad para levantar el fluido necesario a la superficie. Nuevamente el punto de intersección de la curva del sistema con cualquiera de las curvas a diferentes velocidades es el punto óptimo de operación, esto se puede apreciar en la figura 5. 
Edison R. Suntaxi-Guanotoa; Rene A. Carrillo-Flores; Iván C. Naula-Reina; Carlos F. Izurieta-Cabrera; Guillermo A. AlbujaProaño; Darwin R. Caina-Aysabucha

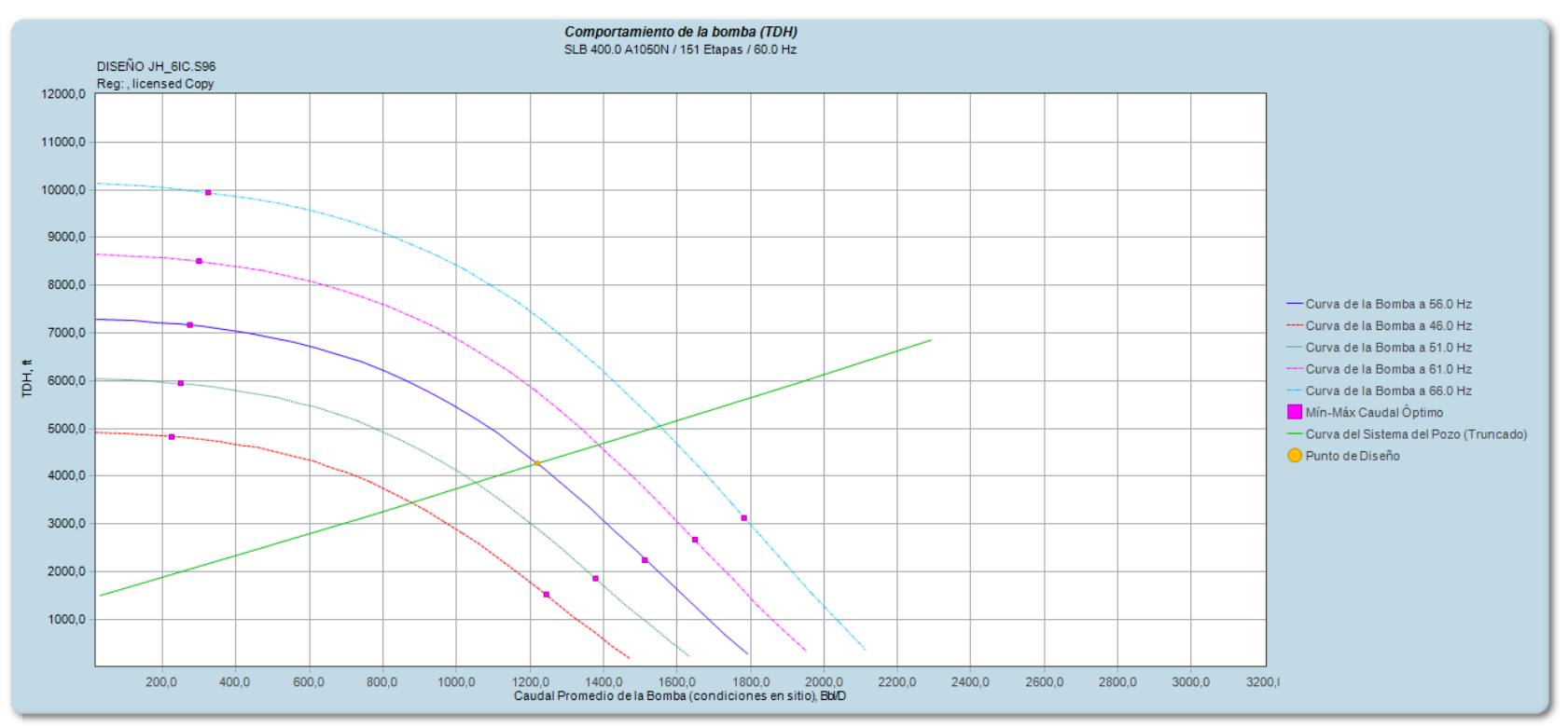

Figura 5. Curva de la bomba multi-frecuencia diseño original

(Derechos de Autor - Resultados de Análisis SubPUMP)

El resultado final del diseño se lo obtiene cuando está optimizado todo el sistema, realizando un análisis técnico económico para ver cuál de las alternativas es la mejor, a lo cual hace referencia la figura 6 y 7. 
Determinación y Optimización del punto de operación de las bombas del sistema electro-sumergible para pozos petroleros

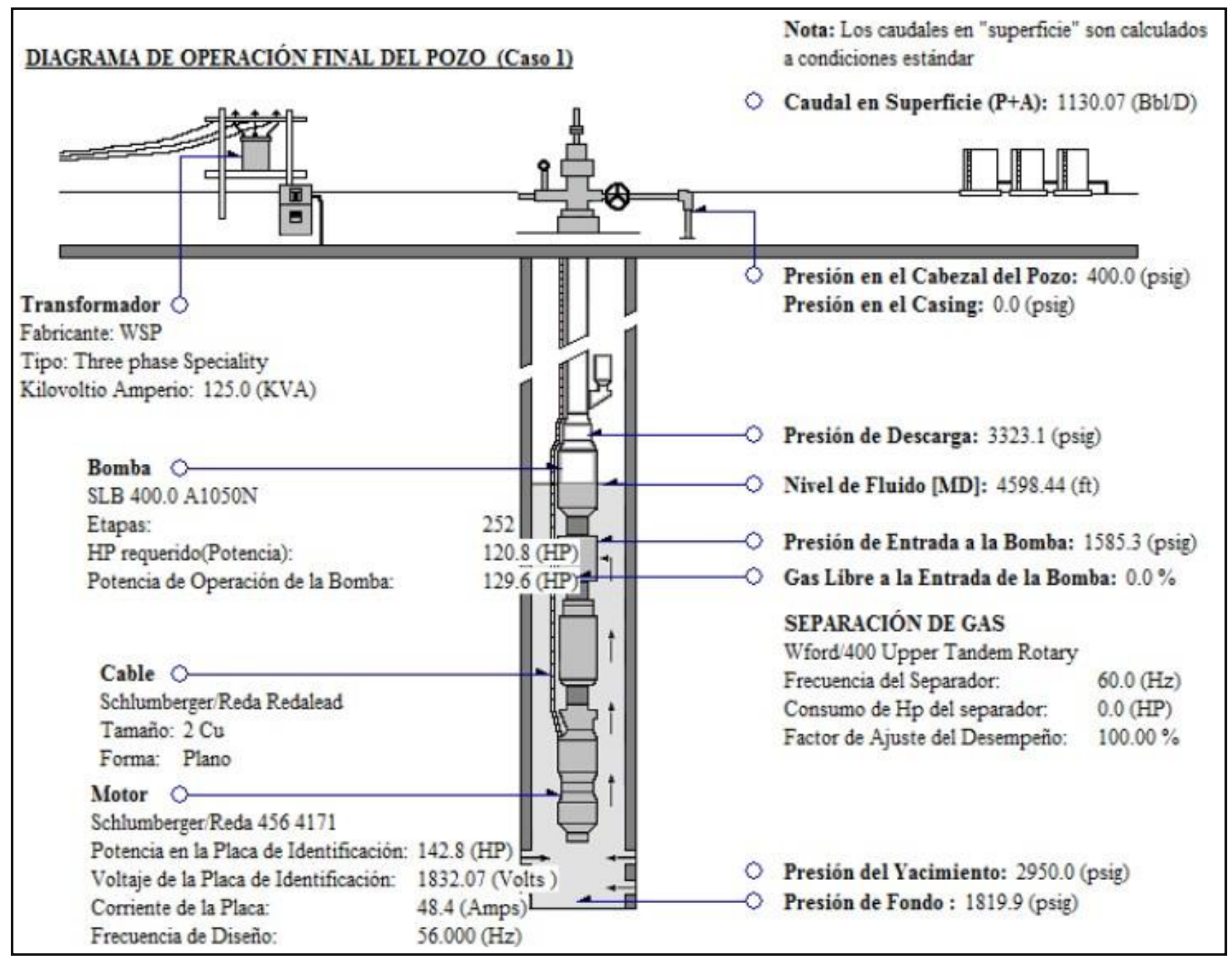

Figura 6. Diagrama final y parámetros de operación diseño inicial 
Edison R. Suntaxi-Guanotoa; Rene A. Carrillo-Flores; Iván C. Naula-Reina; Carlos F. Izurieta-Cabrera; Guillermo A. AlbujaProaño; Darwin R. Caina-Aysabucha

\begin{tabular}{|c|c|c|}
\hline \multicolumn{3}{|l|}{ OPERACIÓN Y COMPORTAMIIENTO } \\
\hline Caso: & (1) & (2) \\
\hline Frecuencia, $\mathrm{Hz}:$ & 56.000 & 45.000 \\
\hline HP requerido, HP: & 120.8 & 101.5 \\
\hline Carga de operación del Motor, \%: & 84.6 & 71.1 \\
\hline Velocidad de Operación, RPM: & 3227.1 & 2593.2 \\
\hline Corriente de Operación, Amps: & 49.1 & 32.9 \\
\hline Voltaje de Operación, Volts : & 1709.9 & 1374.0 \\
\hline Factor de Potencia de Operación : & 0.794 & 0.638 \\
\hline \multicolumn{3}{|l|}{ RPM del Motor Ajustadas por Deslizamiento: } \\
\hline & $\mathrm{Si}$ & $\mathrm{Si}$ \\
\hline Efic. de la Bomba, \%: & 26.7 & 33.5 \\
\hline Eficiencia del Motor, \%: & 83.8 & 83.8 \\
\hline Carga de Empuje de Operación, lb: & N/A & N/A \\
\hline Carga de Empuje Máxima, lb: & $\mathrm{N} / \mathrm{A}$ & N/A \\
\hline Caudal de Líq. Final en Sup(P+A), BblD: & 1130.07 & 780.62 \\
\hline Total de Etapas : & 252 & 252 \\
\hline \multicolumn{3}{|l|}{ Prom. Final de Fluido en la Bomba, Bbl/D: } \\
\hline & 1219.93 & 842.69 \\
\hline Caudal Total Prom. de la Bomba, Bbl/D: & 1243.14 & 862.58 \\
\hline \multicolumn{3}{|l|}{ Volumen de Gas Libre a la entrada de la Bomba, \%: } \\
\hline & 0.0 & 0.0 \\
\hline Volumen de Gas Libre dentro de la Bomba, \%: & 0.0 & 0.0 \\
\hline Cabeza Dinámica Total (TDH), ft: & 4269.26 & 3367.81 \\
\hline Presión de entrada a la Bomba, psig: & 1585.3 & 1934.5 \\
\hline Presión de Descarga, psig: & 3323.1 & 3305.4 \\
\hline Potencia de Operación de la Bomba, HP: & 129.6 & 56.2 \\
\hline Presión de Fondo ,psig: & 1819.9 & 2169.4 \\
\hline Nivel de Fluido [MD], ft: & 4598.44 & 3387.68 \\
\hline
\end{tabular}

Figura 7. Resultados del diseño inicial

(Derechos de Autor - Resultados de Análisis SubPUMP)

\section{Análisis de sensibilidad.}

Una vez realizada la instalación del equipo las condiciones del pozo durante su vida productiva van cambiando, por ende se requiere realizar un análisis de sensibilidad variando las condiciones operativas que más afecta al sistema, lo cual se puede apreciar en la figura 8 y 9. 
Determinación y Optimización del punto de operación de las bombas del sistema electro-sumergible para pozos petroleros

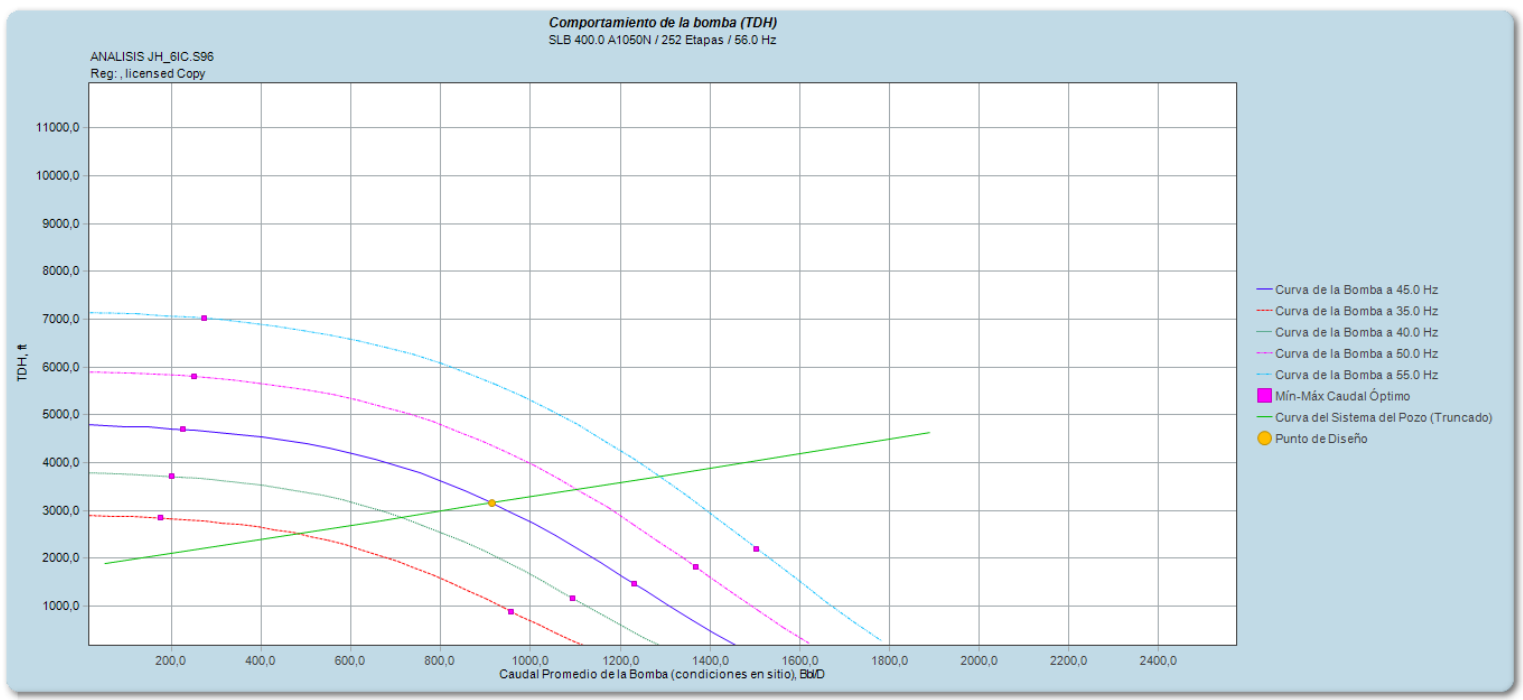

Figura 8. Curva de la bomba multi-frecuencia

(Derechos de Autor - Resultados de Análisis SubPUMP)

\begin{tabular}{|c|c|c|c|c|}
\hline \multicolumn{5}{|l|}{ OPERACIÓN Y COMPORTAMIIENTO } \\
\hline Caso: & (1) & (2) & (3) & (4) \\
\hline Comentarios: & analisis a $45 \mathrm{hz}$ & analisis a $46 \mathrm{hz}$ & analisis a $47 \mathrm{hz}$ & analisis a $56 \mathrm{hz}$ \\
\hline Frecuencia de Diseño, Hz: & 45.000 & 46.000 & 47.000 & 56.000 \\
\hline HP requerido, HP: & 76.8 & 78.3 & 79.9 & 95.8 \\
\hline Carga de operación del Motor, \%: & 51.2 & 52.2 & 53.3 & 63.9 \\
\hline Velocidad de Operación, RPM: & 2613.8 & 2668.4 & 2722.9 & 3201.8 \\
\hline Corriente de Operación, Amps: & 24.5 & 25.7 & 26.9 & 41.2 \\
\hline Voltaje de Operación, Volts : & 1578.8 & 1613.8 & 1648.9 & 1964.7 \\
\hline Factor de Potencia de Operación : & 0.830 & \multicolumn{3}{|c|}{$\begin{array}{l}\text { Factor de Potencia de Operación : } \\
\text { RPM del Motor Ajustadas por Deslizamiento: }\end{array}$} \\
\hline 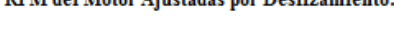 & $\mathrm{Si}$ & $\mathrm{Si}$ & $\mathrm{Si}$ & $\mathrm{Si}$ \\
\hline Efic. de la Bomba, \%: & 31.3 & 30.4 & 29.6 & 23.6 \\
\hline Eficiencia del Motor, \%: & 80.0 & 80.8 & 81.6 & 85.3 \\
\hline Carga de Empuje de Operación, lb: & N/A & $\mathrm{N} / \mathrm{A}$ & N/A & N/A \\
\hline Carga de Empuje Máxima, lb: & N/A & $\mathrm{N} / \mathrm{A}$ & N/A & N/A \\
\hline & 837.66 & 870.99 & 904.07 & 1182.81 \\
\hline Total de Etapas : & 252 & 252 & 252 & 252 \\
\hline \multicolumn{5}{|l|}{ Prom. Final de Fluido en la Bomba, Bbl/D: } \\
\hline \multirow{2}{*}{\multicolumn{5}{|c|}{ Caudal Total Prom. de la Bomba, BblD: }} \\
\hline & & & & \\
\hline & 921.97 & 960.66 & 999.22 & 1330.40 \\
\hline \multicolumn{5}{|c|}{ Volumen de Gas Libre a la entrada de la Bomba, \%: } \\
\hline & 8.0 & 7.8 & 7.6 & 6.4 \\
\hline Volumen de Gas Libre dentro de la Bomba, : & 0.0 & 0.0 & 0.0 & 0.0 \\
\hline Cabeza Dinámica Total (TDH), ft: & 3162.42 & 3217.40 & 3270.36 & 3725.49 \\
\hline Presión de entrada a la Bomba, psig: & 2203.9 & 2183.4 & 2163.0 & 1991.4 \\
\hline Presión de Descarga, psig: & 3467.0 & 3468.5 & 3469.3 & 3479.5 \\
\hline Potencia de Operación de la Bomba, HP: & 60.2 & 65.4 & 70.9 & 132.4 \\
\hline Presión de Fondo , psig: & 2429.7 & 2409.0 & 2388.5 & 2215.3 \\
\hline Nivel de Fluido [MD], ft: & 2293.19 & 2365.69 & 2437.64 & 3043.95 \\
\hline
\end{tabular}

Figura 9. Resultados del análisis de sensibilidad

(Derechos de Autor - Resultados de Análisis SubPUMP) 


\section{Optimización.}

Se la utiliza cuando se han instalado sensores de fondo de pozo en un pozo con ESP, y los datos muestreados a partir de estos sensores se cotejan con una evaluación de ESP para determinar la exactitud de las evaluaciones. Los datos medidos, tales como frecuencia de operación, caudal en superficie, valores de presión y temperatura del cabezal del pozo, PIP, y descarga de la bomba, se sustituirán en una evaluación activa del programa SubPUMP, y se utilizarán para efectuar un cálculo especial de optimización de SubPUMP. La precisión del cálculo de optimización y la información de diagnóstico se pueden mejorar maximizando los elementos de datos medidos disponibles utilizados como sustituciones.

Los datos medidos disponibles para la presión de fondo de flujo, presión de entrada de la bomba y presión de descarga de la bomba se comparan con las presiones calculadas de SubPUMP y se reportan los porcentajes de diferencia correspondientes.

Para un pozo ESP típico diseñado con SubPUMP, se espera que los datos medidos se aproximen a los resultados del programa, lo cual indica que la ESP está funcionando en condiciones óptimas. Si se presenta alguna discrepancia en los resultados, significa que hay un problema en el pozo. La herramienta de Optimización está diseñada para ayudar a los técnicos a investigar (o diagnosticar) un pozo ESP mediante la interpretación hidráulica, lo mencionado anteriormente se puede referenciar en la figura 10. 
Determinación y Optimización del punto de operación de las bombas del sistema electro-sumergible para pozos petroleros

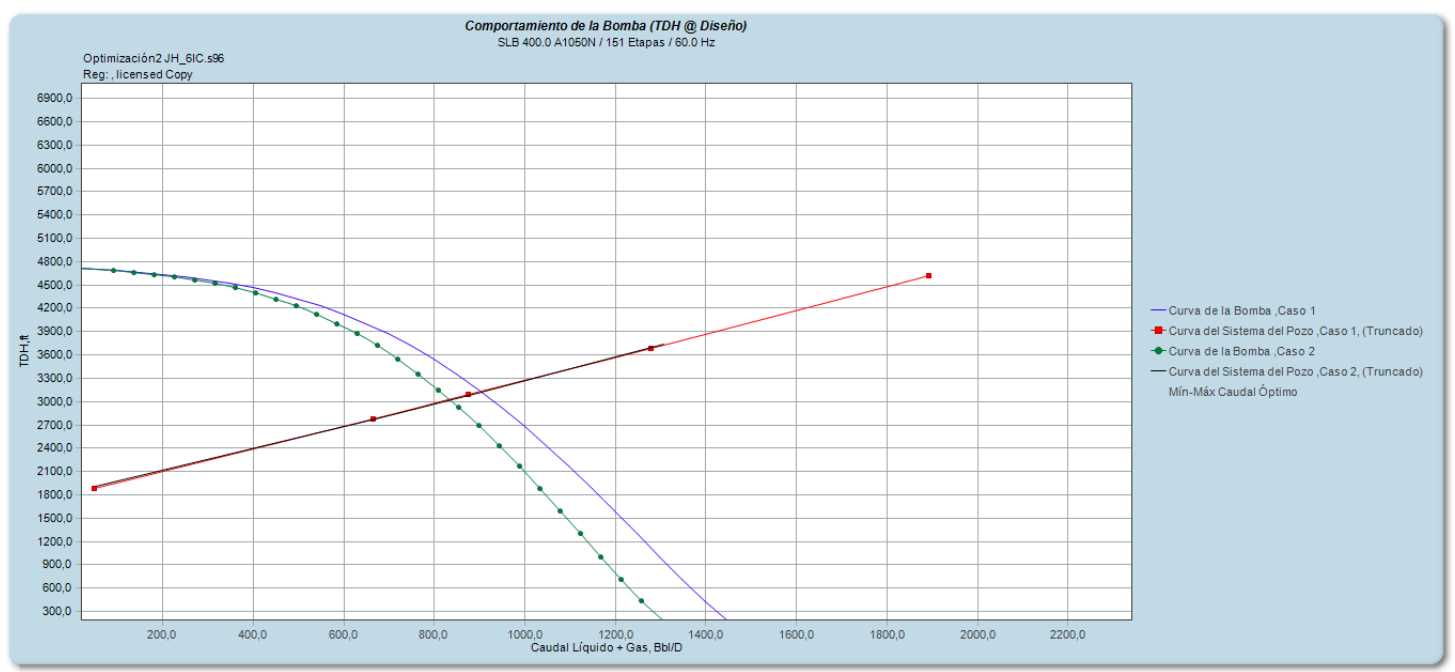

Figura 10. Curva de la bomba optimizada el corte de agua

(Derechos de Autor - Resultados de Análisis SubPUMP)

\section{Conclusiones.}

En la tabla 1, podemos observar que el error entre el diseño original del equipo ESP y los datos de operación después que fue instalado el equipo ESP es de $4.7 \%$ referente al caudal y $16.3 \%$ referente a la presión de entrada de la bomba PIP.

Tabla 1. Error relativo del diseño vs medido

(Derechos de Autor - Resultados de Análisis)

\begin{tabular}{|l|l|l|l|}
\hline \multicolumn{4}{|l|}{ Error relativo del diseño vs medido } \\
\hline $\mathrm{Hz}$ & Qbomba (bfpd) & PIP (psi) & PDB (psi) \\
\hline 56 & $-3.7 \%$ & $-20.4 \%$ & $-4.5 \%$ \\
\hline 45 & $-57 \%$ & $-12.3 \%$ & $-4.7 \%$ \\
\hline Promedio & $\mathbf{- 4 . 7 \%}$ & $\mathbf{- 1 6 . 3 \%}$ & $\mathbf{- 4 . 6 \%}$ \\
\hline
\end{tabular}


Esto es debido a que los parámetros iniciales de diseño son diferentes con los parámetros actuales de operación, los más significativos son índice de producción del pozo IP y la densidad del fluido API.

Después que un pozo se encuentra en operación normal, ajustando los únicos parámetros frecuencia y presión de cabeza del pozo, si es que amerita, procedemos a tomar las lecturas de la presión de entrada a la bomba PIP, presión de descarga de la bomba PDB y la prueba de producción del pozo, para realizar la optimización del sistema. El error relativo de la presión de entrada a la bomba es $1.36 \%$ y la presión de descarga de $2.74 \%$.

\section{Tabla 2. Error relativo de parámetros críticos para optimización de la bomba}

\section{(Derechos de Autor - Resultados de Análisis)}

\begin{tabular}{|l|l|l|l|}
\hline \multicolumn{4}{|c|}{ Parámetros críticos para la optimización de bombas ESP } \\
\hline & Calculado & Medido & Error \\
\hline Presión de entrada a la bomba & 2225.84 & 2196 & $1.36 \%$ \\
\hline Presión de descarga de la bomba & 3390.36 & 3300 & $2.74 \%$ \\
\hline
\end{tabular}

De los resultados obtenidos mediante el análisis y simulación del diseño del sistema de bombeo electro-sumergible de un pozo petrolero mediante la utilización del programa SubPUMP, se constata que el equipo está limitado a un caudal de producción y a una columna dinámica generada fija, los cuales cambian variando la velocidad del equipo sin necesidad de modificar el mismo facilitando en algunos casos si los datos del pozo no difieren mucho del diseño a optimizar el funcionamiento del sistema electro-sumergible. 
Determinación y Optimización del punto de operación de las bombas del sistema electro-sumergible para pozos petroleros

Con el análisis y pruebas realizadas una vez estabilizada la operación de la bomba y con los datos de parámetros medidos en las pruebas de producción se observó que si se puede optimizar el funcionamiento de la bomba.

\section{Bibliografía.}

Baker Hughes. (2009). Submersible Pump Handbook, $9^{\text {th }}$. Edition. Claremore, USA: Centrilift.

Beggs, H. (2003). Production Optimization: Using NODAL ${ }^{\mathrm{TM}}$ Analysis. Tulsa, Oklahoma: OGCI Publications.

Instipetrol. (2017). Instituto de Automatización Petrolera: Producción I, Bombeo Electrosumergible Bombas Centrífugas. Recuperado de: https://es.slideshare.net/daviddesing/bombeo-electrosumergible-bombascentrifugas.

Gabor, T. (2009). Electrical submersible pumps manual: Design, operations and Maintenance. BurlingtonMassachusetts: El Servier.

Maggiolo, R. (2008). ESP oil international training group: Workshop international. "Optimización de la producción mediante Análisis Nodal". Lima-Perú.

Oilproduction. (2017). Análisis de un Sistema de Producción y Análisis Nodal. Recuperado de: http://oilproduction.net/files/analisis_nodal_hirschfeldt.pdf.

Sachdeva, R. (1988). Two-Phase flow through Electric Submersible Pumps, PhD dissertation, The University of Tulsa.

SubPUMP. Technical Reference Manual. 